\title{
A DECOMPOSITION THEOREM FOR $n$-DIMENSIONAL MANIFOLDS
}

\author{
P. H. DOYLE AND J. G. HOCKING
}

Throughout our discussion an $\boldsymbol{n}$-dimensional manifold will mean a connected, separable metric space in which each point has an open $n$-cell neighborhood. Our main result can be stated in the following manner.

THEOREM 1. Let $M^{n}$ be an $n$-dimensional manifold. Then $M^{n}=P^{n} \cup C$, where $P^{n}$ is homeomorphic to euclidean $n$-space, $E^{n}$, and $C$ is a closed subset of $M^{n}$ of dimension at most $n-1$; and $P^{n} \cap C=\square$.

Considered from one point of view Theorem 1 is a generalization of Corollary 1 in [3]. From still another the result says that any $n$-manifold is "almost triangulable." The proof of Theorem 1 leads to more interesting results in the case of compact manifolds which we shall consider presently.

The steps in the proof will be described here. If $C^{n}$ is a closed $n$-cell in $M^{n}$ such that $\mathrm{Bd} C^{n}$, the boundary of $C^{n}$, is bicollared in $M^{n},[2]$, and if $\left\{a_{i}\right\}$ is a countable dense subset of $M^{n} \backslash C^{n}$, consider the set $C^{n} \cup a_{1}$. Does this set lie on the interior of an $n$-cell in $M^{n}$ with a bicollared boundary? If this were the case and if $C_{1}$ is such an $n$-cell, one could ask if $C_{1} \cup a_{2}$ lies interior to an $n$-cell in $M^{n}$ with a bicollared boundary. Continuing in this way with sets of the form $C_{i} \cup a_{i+1}$, if such enclosure is always possible, we obtain an increasing sequence $\left\{C_{i}\right\}$ of closed $n$-cells in $M^{n}, \mathrm{Bd} C_{i}$ is bicollared in $M^{n}$ and int $C_{i+1}$ $\supset C_{i}$, where interior $C_{i+1}$ is written int $C_{i+1}$. Next we observe that $P^{n}=U_{i} C_{i}$ is $E^{n}$ by either a direct construction of cells with annuli between them or by applying the main result of [1]. Then $M^{n}-P^{n}$ $=C$ is nowhere dense in $M^{n}$ and closed since $P^{n}$ is open. The sets $P^{n}$ and $C$ would then meet the requirements of Theorem 1.

From this outline it is clear that the proof of Theorem 1 follows immediately from a lemma.

Lemma 1. Let $M^{n}$ be an $n$-manifold and $D^{n}$ a closed $n$-cell in $M^{n}$ with bicollared boundary. Then if $p$ is any point in $M^{n}, D^{n} \cup_{p}$ lies in int $D_{1}^{*}$, where $D_{1}^{n}$ is a closed $n$-cell and $\mathrm{Bd} D_{1}^{n}$ is bicollared.

Proof. Let $q$ be any point in int $D^{n}$. There is a homeomorphism $h$ of $M^{n}$ onto $M^{n}$ which is pointwise fixed outside any neighborhood $V$ of $D^{n}$ and which carries $D^{n}$ into any preassigned neighborhood $u$ of $q$

Received by the editors May 11, 1961. 
while $h(q)=q$. This follows from the fact that $\mathrm{Bd} D^{n}$ is bicollared.

Since $M^{n}$ is a manifold, the set $p \cup q$ lies interior to an $n$-cell in $M^{n}$ and so interior to an $n$-cell with a bicollared boundary in $M^{n}$. Evidently we need only select $\mathfrak{u}$ in the interior of such a cell to obtain the proof of Lemma 1.

With the proof of Lemma 1 we obtain Theorem 1 . In the case $M^{n}$ is compact the conditions on $C$ are stronger.

Theorem 2. Let $M^{n}$ be a compact $n$-dimensional manifold. Then $M^{n}=P^{n} \cup C$, where $P^{n}$ is homeomorphic to $E^{n}$, and $C$ is a nonseparating continuum in $M^{n} ; P^{n} \cap C=\square$.

It is convenient to call the decomposition $P^{n} \cup C$ of $M^{n}$ in the above theorems a standard decomposition if $P^{n}$ is obtained as in the proof of Theorem 1.

COROLlaRY 1. Let $M^{n}$ be a compact $n$-manifold and $M^{n}=P^{n} \cup C a$ standard decomposition of $M^{n}$. Then if there is a homeomorphism $h$ of $M^{n}$ onto $M^{n}$ such that $h(C) \subset P^{n}$, then $M^{n}$ is an $n$-sphere.

Proof. By Theorem 2, $C$ is compact and so $h(C)$ lies in the interior of a closed $n$-cell $C^{n}$ in $P^{n}$. By the construction of $P^{n}, M^{n}$ is the union of two closed $n$-cells with no boundary points in common. Whence, as in Lemma 3 of [4], one can conclude that $M^{n}$ is a sphere.

CoROllaRY 2. Let $M^{n}$ be a compact $n$-compact $n$-manifold and let $M^{n}=P_{1}^{n} \cup C_{1}=P_{2}^{n} \cup C_{2}$ be two standard decompositions. If $C_{1} \cap C_{2}=\square$, then $M^{n}$ is a sphere.

It should be pointed out that the set $C$ in a standard decomposition need not be nice. In the case of the 2 -sphere $S^{2}, C$ may be any nonseparating 1-dimensional continuum in $S^{2}$; so $C$ need not be locally connected.

Theorem 3. Let $M^{n}$ be an $n$-manifold and $S^{n}$, the $n$-sphere. Then there is a map $f$ from $M^{n}$ onto $S^{n}$ such that each point of $S^{n}$ has a degenerate inverse except perhaps for one point $p$, and $\operatorname{dim} f^{-1}(p) \leqq n-1$.

Proof. The representation of $P^{n}$ as an increasing sequence of $n$ cells provides an evident map of the type described with $C$ as the only possible nondegenerate inverse. In case $M^{n}=E^{n}, C$ may be void; however, one may arrange it so that $C$ is not void even in this case.

In the proof of Corollary 1 to Theorem 2 we observed that a compact $n$-manifold which fails to be a sphere cannot be the union of two closed $n$-cells having no boundary points in common. Similar results 
may be obtained for open regions. If $M^{n}$ is a compact $n$-manifold, $P^{n} \cup C=M^{n}$, a standard decomposition, let $C$ be in an open set $u$ in $M^{n}$ such that $\mathcal{U}$ is homeomorphic to a subset of $E^{n}$. Then if $h$ is an imbedding of $\mathcal{u}$ in $E^{n}$ we note that $h(C)$ is the limit in $E^{n}$ of a strictly decreasing sequence of closed $n$-cells with bicollared boundaries in $h(\mathcal{U}) \subset E^{n}$. Whence, we obtain $M^{n}$ as a union of closed $n$-cells with disjoint bicollared boundaries and so $M^{n}$ is an $n$-sphere. We can then assert another theorem.

THEOREM 4. If $M^{n}$ is a compact n-manifold which is not an $n$-sphere and if $M^{n}=P^{n} \cup C$ is a standard decomposition, then $C$ has no neighborhood in $M^{n}$ which can be imbedded in $E^{n}$.

\section{REFERENCES}

1. M. Brown, The monotone union of open n-cells is an open n-cell, Proc. Amer. Math. Soc. 12 (1961), 812-814.

2. M. Brown and E. Michael, Collared subsets of metric spaces, Abstract 576-128, Notices Amer. Math. Soc. 7 (1960), 940.

3. M. K. Fort, Jr., A theorem about topological n-cells, Proc. Amer. Math. Soc. 5 (1954), 456-459.

4. J. R. Stallings, Polyhedral homotopy-spheres, Bull. Amer. Math. Soc. 66 (1960), 485-488.

Michigan State University 